\title{
Microbiota metabolite short-chain fatty acid acetate promotes intestinal IgA response to microbiota which is mediated by GPR43
}

\author{
$\mathrm{W}$ Wu ${ }^{1,2,5}, \mathrm{MSun}^{1,2,5}, \mathrm{FChen}^{3}, \mathrm{AT} \mathrm{Cao}^{2}, \mathrm{H} \mathrm{Liu}^{2}, \mathrm{Y} \mathrm{Zhao}^{2}, \mathrm{X} \mathrm{Huang}^{2}, \mathrm{Y} \mathrm{Xiao}^{2}, \mathrm{~S} \mathrm{Yao}^{2}, \mathrm{Q}^{\mathrm{Zhao}}{ }^{4}, \mathrm{Z} \mathrm{Liu}^{1}$ and \\ Y Cong ${ }^{2,3}$
}

Intestinal IgA, which is regulated by gut microbiota, has a crucial role in maintenance of intestinal homeostasis and in protecting the intestines from inflammation. However, the means by which microbiota promotes intestinal lgA responses remain unclear. Emerging evidence suggests that the host can sense gut bacterial metabolites in addition to pathogenassociated molecular patterns and that recognition of these small molecules influences host immune response in the intestines and beyond. We reported here that microbiota metabolite short-chain fatty acid acetate promoted intestinal IgA responses, which was mediated by "metabolite-sensing" GPR43. GPR43 ${ }^{-1}$ mice demonstrated lower levels of intestinal $\lg A$ and $\lg \mathrm{A}^{+}$gut bacteria compared with those in wild type (WT) mice. Feeding WT but not GPR43 ${ }^{-{ }^{-}}$mice acetate but not butyrate promoted intestinal IgA response independent of T cells. Acetate promoted B-cell IgA class switching and IgA production in vitro in the presence of WT but not GPR43 ${ }^{-1-}$ dendritic cells (DCs). Mechanistically, acetate-induced DC expression of Aldh1a2, which converts Vitamin A into its metabolite retinoic acid (RA). Moreover, blockade of RA signaling inhibited the acetate induction of B-cell IgA production. Our studies thus identified a new pathway by which microbiota promotes intestinal IgA response through its metabolites.

\section{INTRODUCTION}

The intestinal mucosa establishes state of hypo-responsiveness against commensal bacteria and of active readiness against pathogens. ${ }^{1}$ Despite enormous challenges by the microbiota, the intestine lives in harmony with it, in part due to interactions of the microbiota with the host to maintain intestinal homeostasis. ${ }^{2}$ Multiple host mechanisms have evolved to regulate this relationship. One of the important strategies to generate immune protection and maintain intestinal homeostasis is the production of IgA, the most abundant antibody isotype in the host, which provides a first line of immune protection at the mucosal surface. ${ }^{3-5}$ IgA regulates the microbiota, and gut bacteria, in turn, adapt to IgA by altering their gene expression patterns. ${ }^{6,7}$ Several recent studies have shown that IgA binds colitogenic members of the microbiota, ${ }^{8,9}$ and that mice deficient in IgA or polymeric Ig receptor (pIgR), the epithelial cell receptor for exporting IgA into the lumen, develop more severe colitis following inflammatory insults. ${ }^{10}$ The findings further the importance of intestinal IgA in the regulation of microbiota-induced inflammatory disease. However, in spite of recent advances, the function and regulation of intestinal IgA remain poorly understood.

The microbiota has a major impact on many host systems, particularly on the development of the intestines and the immune system. The critical role of gut microbiota has long been well established in the regulation of IgA production in the intestinal mucosa, as intestinal IgA-secreting cells and IgA production are almost absent in germ-free (GF) animals and rapidly induced by the presence of commensal bacteria, ${ }^{11,12}$ which is consistent with its major role in host protection at the mucosal-luminal interface. ${ }^{6}$ Multiple signals, including T-cell-dependent and -independent pathways, regulate IgA

${ }^{1}$ Department of Gastroenterology, The Shanghai Tenth People's Hospital, Tongji University, Shanghai, China. ${ }^{2}$ Department of Microbiology and Immunology, University of Texas Medical Branch, Galveston, TX, USA. ${ }^{3}$ Department of Pathology, University of Texas Medical Branch, Galveston, TX, USA and ${ }^{4}$ Bristol-Myers Squibb, Princeton, NJ, USA. Correspondence: Z Liu or Y Cong (liuzhanju88@126.com or yicong@utmb.edu)

${ }^{5}$ These authors contributed equally to this work

Received 17 September 2016; accepted 28 October 2016; published online 14 December 2016. doi:10.1038/mi.2016.114 
induction. ${ }^{13} \mathrm{~A}$ role for microbial signals via Toll-like receptors (TLRs) has been reported in mediating intestinal epithelial cell (IEC) and dendritic cell (DC) induction of the production of IgA through the induction of BAFF and APRIL. ${ }^{14,15}$ Furthermore, IEC and T-cell expression of MyD88, which mediates most TLR pathways, promotes B-cell IgA production. ${ }^{14,16}$ However, under steady-state conditions, lack of TLR signaling in $\mathrm{MyD} 88^{-1-}$ mice results in more intestinal IgA production compared with that in WT mice after colonization with commensal bacteria, which has been considered as a mechanism functionally compensating for innate immune deficiency in the clearance of invading microbiota. ${ }^{17}$ Thus, the components of the microbiota critically responsible for regulating intestinal IgA response are still not completely clear.

Emerging evidence indicates the host immune system can sense gut bacterial metabolites in addition to pathogenassociated molecular patterns (PAMP) and that recognition of these small molecules can influence the host immune response in the gut and beyond. ${ }^{18-20}$ Of particular interest are short-chain fatty acids (SCFA), which are solely metabolized by gut bacteria from otherwise indigestible carbohydrates of fiberrich diets, ${ }^{21}$ and have been shown to ameliorate disease in animal models of colitis and allergic asthma. ${ }^{20,22}$ Acetate, propionate and butyrate are the most abundant SCFA. Their collective concentrations in colonic lumen in humans range from 50 to $150 \mathrm{mM}^{21}$ While the exact mechanisms for the action of SCFA are still not completely clear, most notable among the SCFA targets is the "metabolite-sensing" mammalian G protein-coupled receptor pair of GPR41 and GPR43. SCFA can regulate cell function either by inhibiting histone deacetylase activity, thus, affecting gene transcription, or through the activation of GPRs. The dietary fiber has been shown to enhance oral tolerance and induced intestinal IgA response. ${ }^{23}$ A recent report further demonstrated that SCFA promote intestinal IgA responses. ${ }^{24}$ However, the mechanisms by which SCFA regulate intestinal IgA responses are still unknown. In this report, we demonstrated that acetate promoted intestinal IgA responses, which was mediated by GPR43. Mechanistically, acetate induced the DC expression of Aldh1a2, which converts Vitamin A into its metabolite retinoic acid (RA), to promote B-cell IgA production. We thereby identified a new pathway by which microbiota promotes intestinal IgA production through production of metabolite SCFA.

\section{RESULTS}

Intestinal IgA production is decreased in GPR43 ${ }^{-I-}$ mice GPR43 is one of the predominant receptors for SCFA, ${ }^{25}$ and GPR43-SCFA interaction has been implicated in the maintenance of intestinal homeostasis, in that GPR $43^{-/-}$mice develop more severe colitis than do WT mice upon inflammatory insults. ${ }^{26}$ To investigate whether GPR43 regulates intestinal IgA responses, which could contribute to its regulation of intestinal homeostasis, we first determined intestinal IgA production in littermates of WT and
GPR $43^{-/-}$mice. Fecal pellets were collected, and IgA levels determined by ELISA. Fecal IgA production was significantly decreased in GPR $43^{-1}$ mice compared with that in WT mice (Figure 1a). As IgA-coating gut bacteria have been implicated in regulation of intestinal homeostasis, ${ }^{8,9}$ we assessed $\operatorname{IgA}^{+}$gut bacteria in WT and GPR43 $3^{-1-}$ mice. Interestingly, $\operatorname{IgA}{ }^{+}$ bacteria were decreased in GPR43 ${ }^{-1-}$ mice compared with that in WT mice (Figure $\mathbf{1 b}, \mathbf{c}$ ).

The levels of IgA in intestinal lumen are determined by two critical steps: production by B cells and transportation across epithelium to intestinal lumen. Once produced by IgAproducing plasma cells in the mucosa, IgA secretion relies on $\mathrm{pIgR}$, which is expressed on the basolateral surface of epithelial cells, to transport across the intestinal epithelium. ${ }^{27}$ Thus, the levels of IgA in intestinal lumens depend on B-cell IgA production and the rate of $\operatorname{IgA}$ binding to $\mathrm{pIgR}$, which is dictated by the expression levels of the pIgR. ${ }^{27}$ To determine whether GPR43 deficiency affected intestinal epithelial cell (IEC) expression of pIgR, thus contributing to deceased sIgA in the intestines of GPR43 ${ }^{-/-}$mice, we measured pIgR expression in the IEC of WT and GPR $43^{-/-}$mice by quantitative real-time PCR (qRT-PCR). However, comparable levels of pIgR expression were observed in IEC from both WT and GPR $43^{-/-}$mice (Supplementary Fig. S1A), indicating that GPR43 does not affect IEC expression of pIgR. We then investigated IgA-producing B cells in WT and GPR43 ${ }^{-1-}$ mice. In contrast, $\operatorname{IgA}^{+} \mathrm{B}$ cells were significantly decreased in the lamina propria (LP) of GPR43 ${ }^{-/}$mice compared with that in WT mice (Figure 1d,e). However, $\operatorname{IgA}^{+} \mathrm{B}$ cells in spleens and mesenteric lymph nodes (MLN) were observed at similar levels in both WT and GPR $43^{-/-}$mice (Figure 1f,g and Supplementary Figure S1B,C), indicating that GPR43 specifically regulates intestinal mucosal IgA-producing B cells, which is consistent with the presence of high levels of SCFA in the intestinal lumen.

\section{Supplementation with acetate promotes intestinal IgA production in WT but not in GPR43 ${ }^{-I-}$ mice}

To investigate whether SCFA promote intestinal IgA production, we fed acetate, the most potent agonist for GPR43, in drinking water to littermates of WT and GPR43 ${ }^{-1-}$ C57BL/6 mice. As many gut bacteria produce acetate and other SCFA, which are present in the intestines at high levels and could potentially confound the results, we first depleted gut bacteria to decrease gut SCFA by treating mice with broad-spectrum antibiotics for 10 days. The antibiotics treatment depleted about $90 \%$ of gut bacteria (data not shown). Then, a group of antibiotics-treated mice were fed acetate in drinking water containing antibiotics, and a second group received drinking water containing antibiotics only to serve as controls. Fecal pellets were collected 21 days after feeding acetate to determine IgA production. The WT mice treated with acetate exhibited a significant increase in fecal IgA compared with that in control mice (Figure 2a). Fecal IgG production was also measured with no difference between acetate-treated mice and control mice (Figure 2b), indicating that acetate specifically induced IgA 


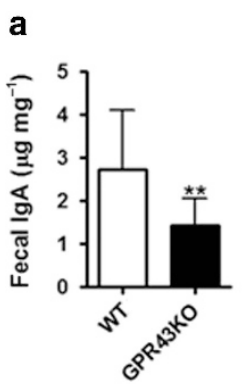

b
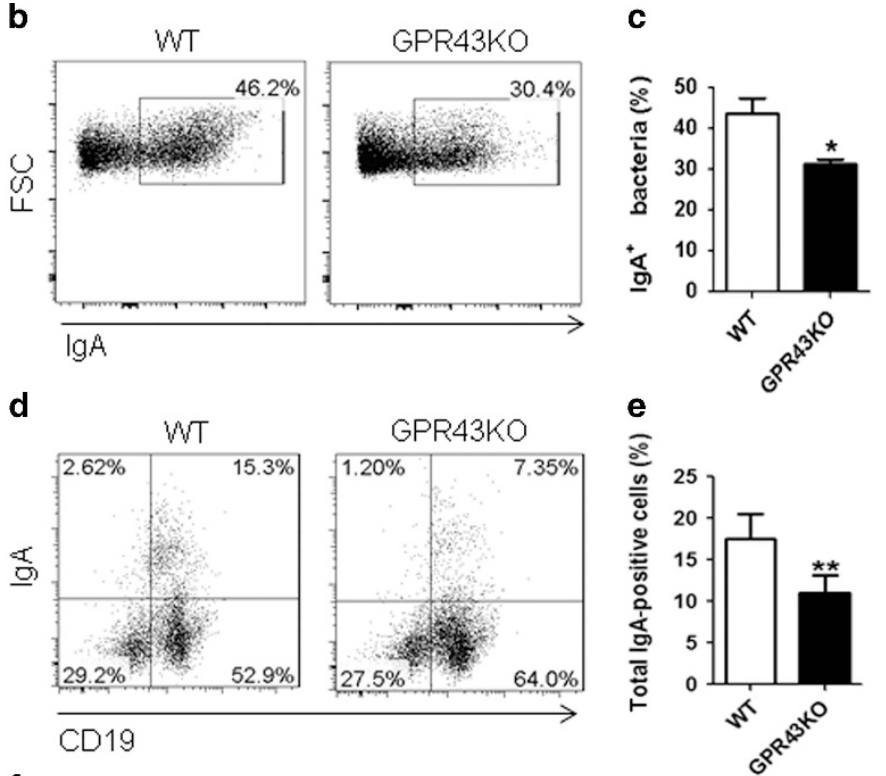

f

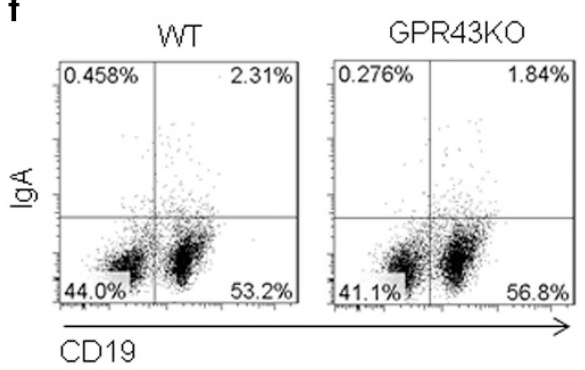

g

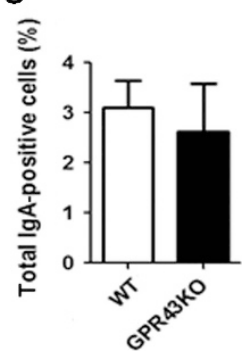

Figure 1 Decreased IgA-producing B cells and IgA production in the intestines of GPR43 ${ }^{-1}$ mice. Fecal pellets were collected from wild type (WT) and

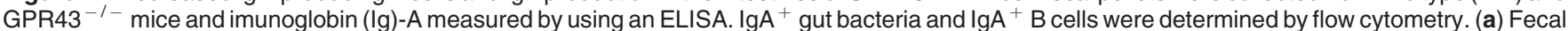
IgA production. Pooled data from 12 mice of 3 experiments. (b) Fluorescence-activated cell sorting (FACS) profiles of IgA ${ }^{+}$gut bacteria. (c) Bar chart of $\operatorname{IgA}{ }^{+}$gut bacteria. (d) FACS profiles of IgA ${ }^{+}$B cells in lamina propria (LP) of the intestines. (e) Bar chart of $\lg A^{+} B$ cells in LP of the intestines of 4 mice per group. (f) FACS profiles of IgA ${ }^{+}$B cells in spleen. (g) Bar chart of $\lg A^{+} B$ cells in the spleens of 4 mice per group. ${ }^{*} P<0.05,{ }^{* *} P<0.01$. The data are representative of three independent experiments.

production in the intestines. The levels of IgA ${ }^{+} \mathrm{B}$ cells were also increased in the intestinal LP of acetate-treated mice compared with those in control mice (Figure 2c,d). However, there was no difference of $\operatorname{Ig} \mathrm{A}^{+} \mathrm{B}$ cells in spleens and MLN between acetate-treated mice and control mice (Figure 2e,f, and Supplementary Figure S2), indicating that acetate specifically induced $\operatorname{IgA}^{+} \mathrm{B}$ cells in the intestinal mucosa. To determine the role of GPR43 in acetate induction of intestinal IgA responses, antibiotics-treated GPR $43^{-1-}$ mice were also fed acetate in drinking water. Interestingly, treatment with acetate did not increase fecal IgA production (Figure 3a) and $\operatorname{IgA}^{+} \mathrm{B}$ cells in LP (Figure $\mathbf{3 b}, \mathbf{c}$ ), spleen, and MLN in GPR $43^{-1-}$ mice (Figure 3d,e, and Supplementary Figure S3). Collectively, these data demonstrated a critical role of GPR43 in acetate induction of intestinal IgA response. To determine whether other SCFA also promoted intestinal IgA production, we fed the antibiotics-treated mice butyrate, relatively weak agonists for GPR43, in drinking water. Fecal $\operatorname{IgA}, \operatorname{IgG}$ and $\operatorname{IgA}^{+} \mathrm{B}$ cells in intestines and spleens were determined 21 days later. Feeding with butyrate did not affect intestinal $\operatorname{IgA}^{+} \mathrm{B}$ cells and fecal IgA (Figure 2a-f and
Supplementary Figure S2). These data indicated that acetate, but not butyrate, promotes intestinal IgA production, which is consistent with their affinity to binding of GPR43, i.e., acetate»butyrate. ${ }^{28}$

\section{T cells are not required for acetate induction of intestinal $\lg \mathrm{A}$}

B-cell IgA production can be regulated by both T-celldependent and -independent pathways. ${ }^{13}$ To investigate whether acetate induction of intestinal IgA requires $\mathrm{T}$ cells, we fed TCR $\beta \times \delta^{-1-}$ mice, which lack T cells but retain $\mathrm{B}$ cells and innate cells intact, with acetate in drinking water for 21 days. Acetate promoted intestinal IgA (Figure 4a) and increased the levels of intestinal LP $\operatorname{IgA}^{+}$B cells in $\operatorname{TCR} \beta \times \delta^{-l-}$ mice (Figure $4 \mathbf{b}, \mathbf{c}$ ), which indicated that acetate induces intestinal IgA production in the absence of T cells. In consistence with WT mice, acetate treatment did not increase the levels of $\operatorname{IgA}{ }^{+} \mathrm{B}$ cells in the spleens of TCR $\beta \mathrm{x} \delta^{-1-}$ mice (Figure 4d,e).

We have previously demonstrated that Th17 cells promoted intestinal IgA responses to microbiota. ${ }^{29}$ To further investigate 

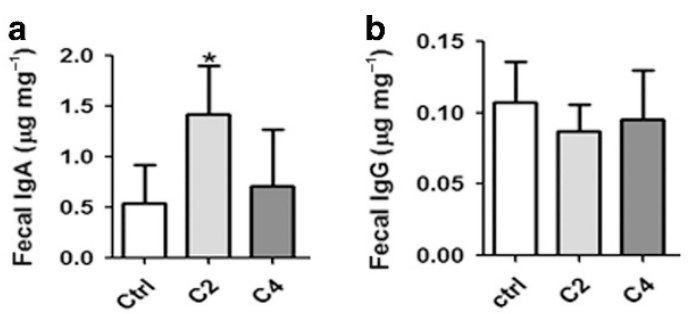

C

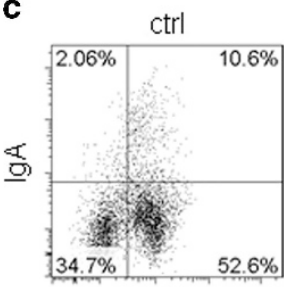

CD19

e

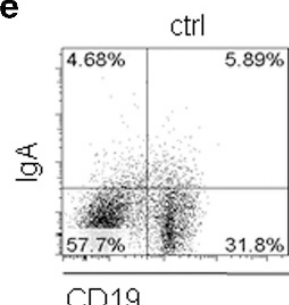

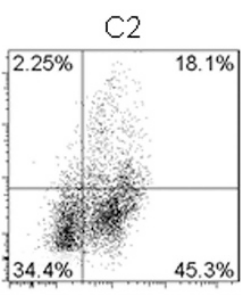

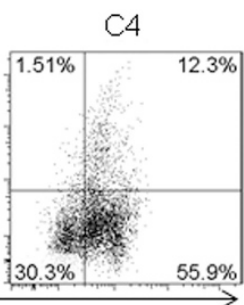

$\stackrel{5.9 \%}{\longrightarrow}$ $130.3 \%$
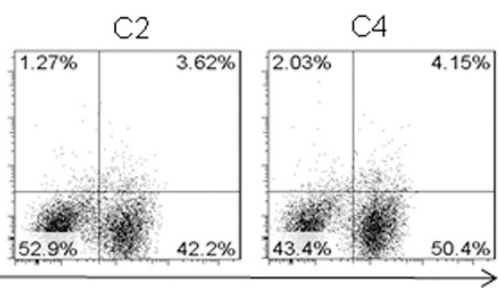

$\stackrel{50.4 \%}{\longrightarrow}$ CD19
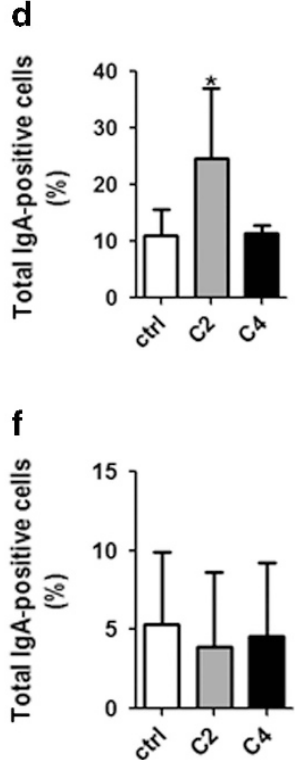

Figure 2 Treatment with acetate promotes $\operatorname{lgA}$-producing B cells and IgA production in intestines. C57BL/6 mice were treated with antibiotics (1 $\mathrm{gl}^{-1}$ metronidazole, $0.5 \mathrm{~g} \mathrm{I}^{-1}$ vancomycin, $1 \mathrm{gl}^{-1}$ ampicillin, and $1 \mathrm{gl}^{-1}$ kanamycin) in drinking water for 10 days, and then fed with or without $300 \mathrm{~mm}$ acetate (C2) or butyrate (C4) in drinking water containing antibiotics for an additional 21 days. Fecal pellets were collected and IgA measured by using an ELISA. $\operatorname{IgA}^{+}$B cells were determined by flow cytometry. (a) Fecal IgA production. (b) Fecal IgG production. (c) Fluorescence-activated cell sorting (FACS) profiles of IgA ${ }^{+} B$ cells in lamina propria (LP) of the intestines. (d) Bar chart of IgA ${ }^{+}$B cells in LP of the intestines of 4 mice per group. (e) FACS profiles of $\operatorname{Ig} A^{+} B$ cells in spleen. (f) Bar chart of $\lg A^{+} B$ cells in the spleens of four mice per group. ${ }^{\star} P<0.05$. The data are representative of two independent experiments.

the role of T-cell expression of GPR43 in the acetate induction of intestinal IgA production, we generated Th17 cells in vitro from WT and GPR43 ${ }^{-1-}$ mice and transferred them into TCR $\beta \times \delta^{-1-}$ mice. Fecal pellets were collected at day 1 prior to $\mathrm{T}$-cell transfer and 4 weeks after T-cell transfer for measuring $\operatorname{IgA} \cdot \operatorname{IgA}^{+} \mathrm{B}$ cells in the spleen and intestines were also determined. Consistent with our previous reports, ${ }^{29}$ fecal IgA was significantly decreased in TCR $\beta \mathrm{x} \delta^{-1-}$ mice compared with that in WT mice (Figure 5a). Transfer of WT and

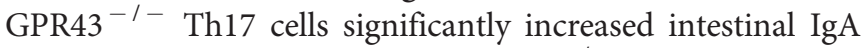
production at similar levels in TCR $\beta \times \delta^{-1-}$ mice (Figure 5a). $\mathrm{IgA}^{+} \mathrm{B}$ cells were also induced in LP, spleen and MLN at similar levels (Figure 5b-e, and Supplementary Figure S4). Taken together, these data demonstrated that $\mathrm{T}$-cell expression of GPR43 does not affect intestinal IgA responses.

\section{Acetate promotes IgA production through DC but not by directly acting on B cells}

As our data indicated that acetate induces intestinal IgA production in the absence of $\mathrm{T}$ cells, we then investigated whether acetate directly acts on B cells to promote IgA production. We isolated splenic IgD ${ }^{+}$naive $\mathrm{B}$ cells and treated them with anti- $\mu$ and CD40L in the presence or absence of acetate for 5-7 days. We also treated B cells with TGF $\beta$, which has been shown to promote B-cell IgA production, ${ }^{13}$ to serve as a positive control. TGF $\beta$ induced B-cell IgA production in the culture. However, acetate treatment did not affect B-cell IgA production (Figure 6a), indicating that acetate did not directly act on $\mathrm{B}$ cells to promote their IgA production. We then asked whether acetate acts in synergy with TGF $\beta$ directly on B cells to induce IgA production. However, addition of acetate did not further enhance B-cell IgA production induced by TGF $\beta$ (Figure 6a).

As DCs have been implicated as being crucial in driving B-cell IgA production, ${ }^{13}$ we asked whether acetate induces B-cell IgA production through regulating DC. We pretreated splenic DCs with acetate for $6 \mathrm{~h}$. After washing to remove free acetate, these acetate-treated DCs were cultured with $\operatorname{IgD}^{+}$ naive B cells for 5-7 days. As shown in Figure 6b, B cells produced significantly higher levels of IgA when cultured with 


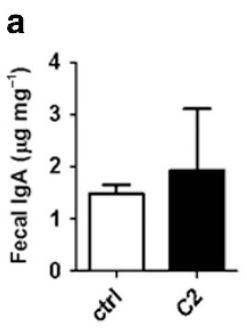

b

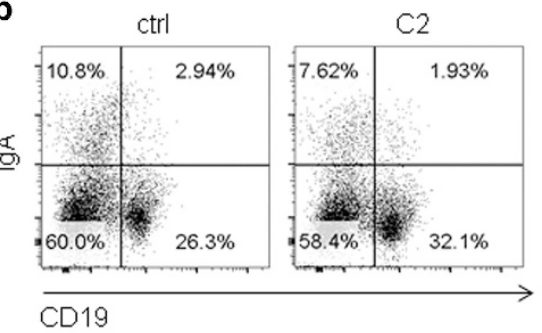

d

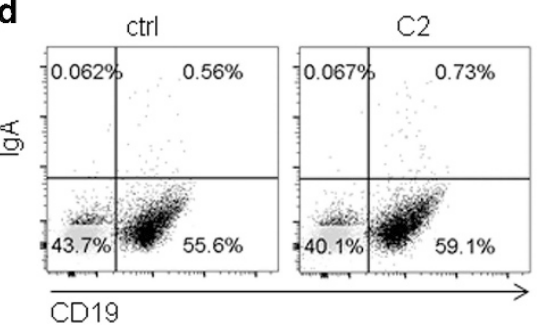

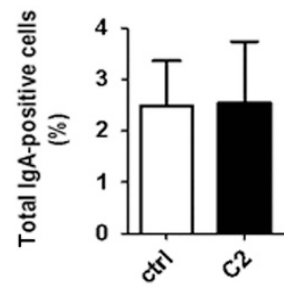

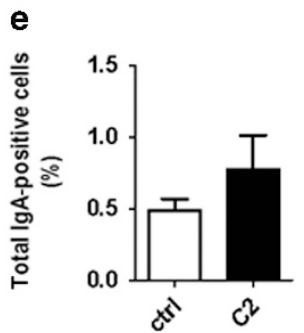

Figure 3 Treatment with acetate did not promote IgA-producing B cells and IgA production in intestines of GPR43 ${ }^{-1-}$ mice. GPR43 ${ }^{-1-}$ mice were treated with antibiotics $\left(1 \mathrm{gl}^{-1}\right.$ metronidazole, $0.5 \mathrm{gl}^{-1}$ vancomycin, $1 \mathrm{gl}^{-1}$ ampicillin, and $1 \mathrm{gl}^{-1}$ kanamycin) in drinking water for 10 days, and then fed with or without $300 \mathrm{~mm}$ acetate (C2) in drinking water containing antibiotics for an additional 21 days. Fecal pellets were collected and IgA measured by using an ELISA. IgA+ B cells were determined by flow cytometry. (a) Fecal IgA production. (b) Fluorescence-activated cell sorting (FACS) profiles of $\operatorname{IgA}{ }^{+}$B cells in lamina propria (LP) of the intestines. (c) Bar chart of IgA ${ }^{+} B$ cells in LP of the intestines of 4-5 mice per group. (d) FACS profiles of IgA ${ }^{+} B$ cells in the spleen. (e) Bar chart of IgA ${ }^{+}$B cells in the spleens of 4-5 mice per group. The data are representative of two independent experiments.

a

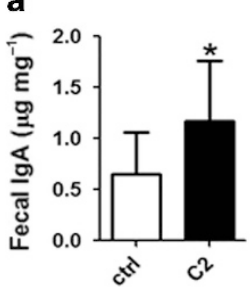

b

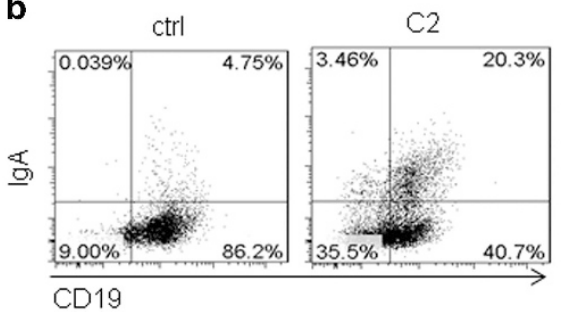

d

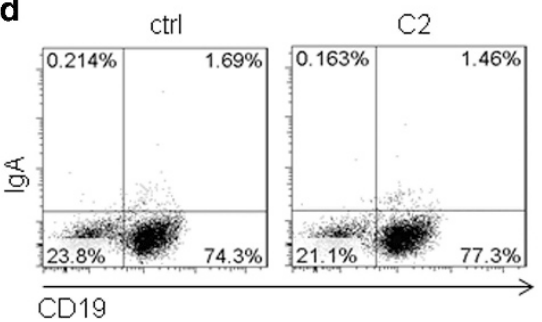

C

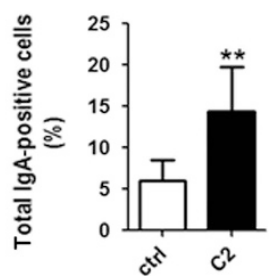

e

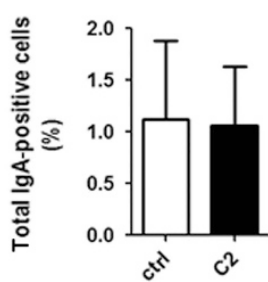

Figure 4 T cells are not required in acetate promotion of IgA-producing B cells and IgA production in intestines. TCR $\beta \times \delta^{-1-}$ mice were treated with antibiotics $\left(1 \mathrm{gl}^{-1}\right.$ metronidazole, $0.5 \mathrm{gl}^{-1}$ vancomycin, $1 \mathrm{gl}^{-1}$ ampicillin, and $1 \mathrm{gl}^{-1}$ kanamycin) in drinking water for 10 days, and then fed with or without $300 \mathrm{~mm}$ acetate (C2) in drinking water containing antibiotics for an additional 21 days. Fecal pellets were collected and IgA measured by using an ELISA. IgA ${ }^{+} B$ cells were determined by using flow cytometry. (a) Fecal lgA production. (b) Fluorescence-activated cell sorting (FACS) profiles of IgA ${ }^{+} B$ cells in lamina propria (LP) of the intestines. (c) Bar chart of IgA ${ }^{+} B$ cells in LP of the intestines of 4 mice per group. (d) FACS profiles of IgA ${ }^{+} B$ cells in the spleen. (e) Bar chart of $\operatorname{IgA}{ }^{+}$B cells in the spleens of $4-5$ mice per group. ${ }^{\star} P<0.05$; ${ }^{\star \star} P<0.01$. The data are representative of two independent experiments.

acetate-treated WT DC compared with those cultured with control DCs. However, acetate-treated GPR43 ${ }^{-1-}$ DC did not affect B-cell IgA production, further confirming the role of acetate binding of DC GPR43 in the induction of B-cell IgA.

In order for B cells to produce antibody, activated B cells must undergo class switch recombination (CSR) through immunoglobulins (Ig) heavy chain locus rearrangement. The Ig recombination is facilitated by the enzyme activation-induced cytokine deaminase $\left(\right.$ Aicda) ${ }^{30}$ Upon stimulation with IgApromoting factors, B cells undergo IgA CSR. These B cells are thus permanently differentiated and can only produce IgA. To determine whether acetate induces CSR, B cells were cultured with acetate-treated DCs, and RNA was collected daily from day 1 to day 4 to determine CSR events. Acetate enhanced B-cell 

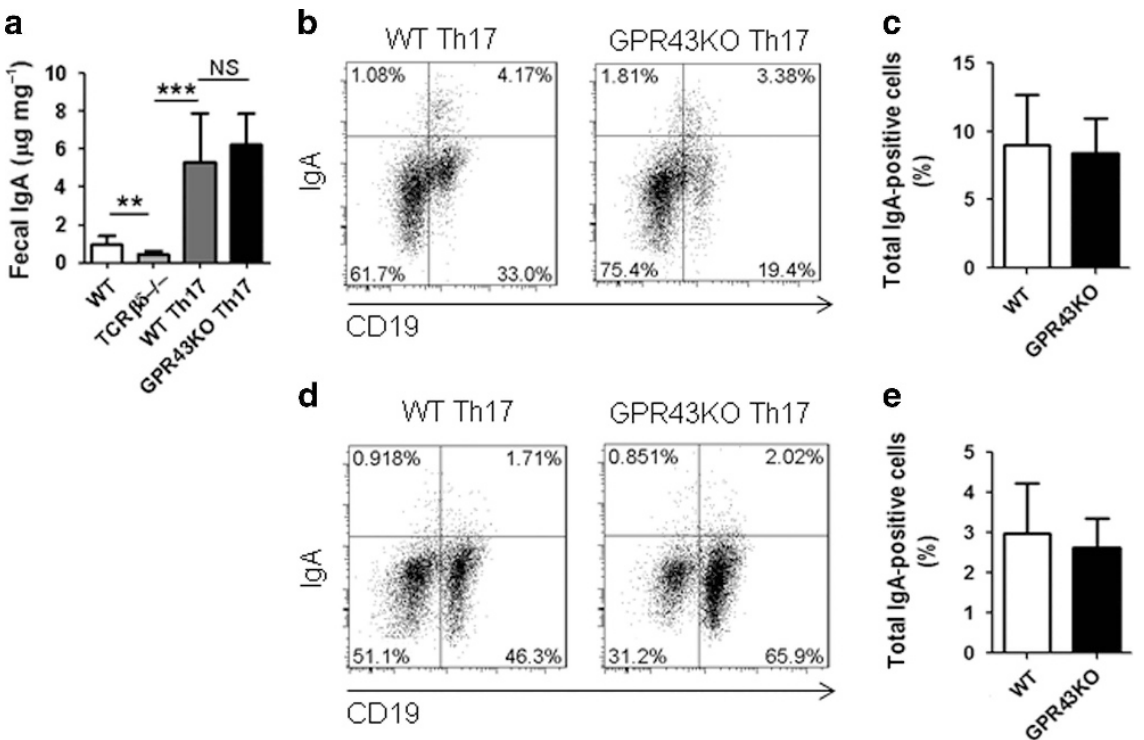

Figure 5 GPR43 ${ }^{-1-}$ T cells induce IgA-producing B cells and IgA production in intestines at levels similar to those in wild type (WT) mice. Th17 cells were generated from WT or GPR $43^{-1-}$ mice, and transferred into TCR $\beta \times \delta^{-1}$ mice. Fecal pellets were collected 4 weeks later and lgA measured by using an ELISA. IgA ${ }^{+}$B cells were determined by using flow cytometry. (a) Fecal IgA production. (b) Fluorescence-activated cell sorting (FACS) profiles of $\lg A^{+} B$ cells in lamina propria (LP) of the intestines. (c) Bar chart of $\lg A^{+} B$ cells in LP of the intestines of 4 mice per group. (d) FACS profiles of IgA ${ }^{+} B$ cells in spleen. (e) Bar chart of IgA ${ }^{+}$B cells in spleens of $4-5$ mice per group. ${ }^{* *} P<0.01 ;{ }^{* * *} P<0.001$. The data are representative of three independent experiments.
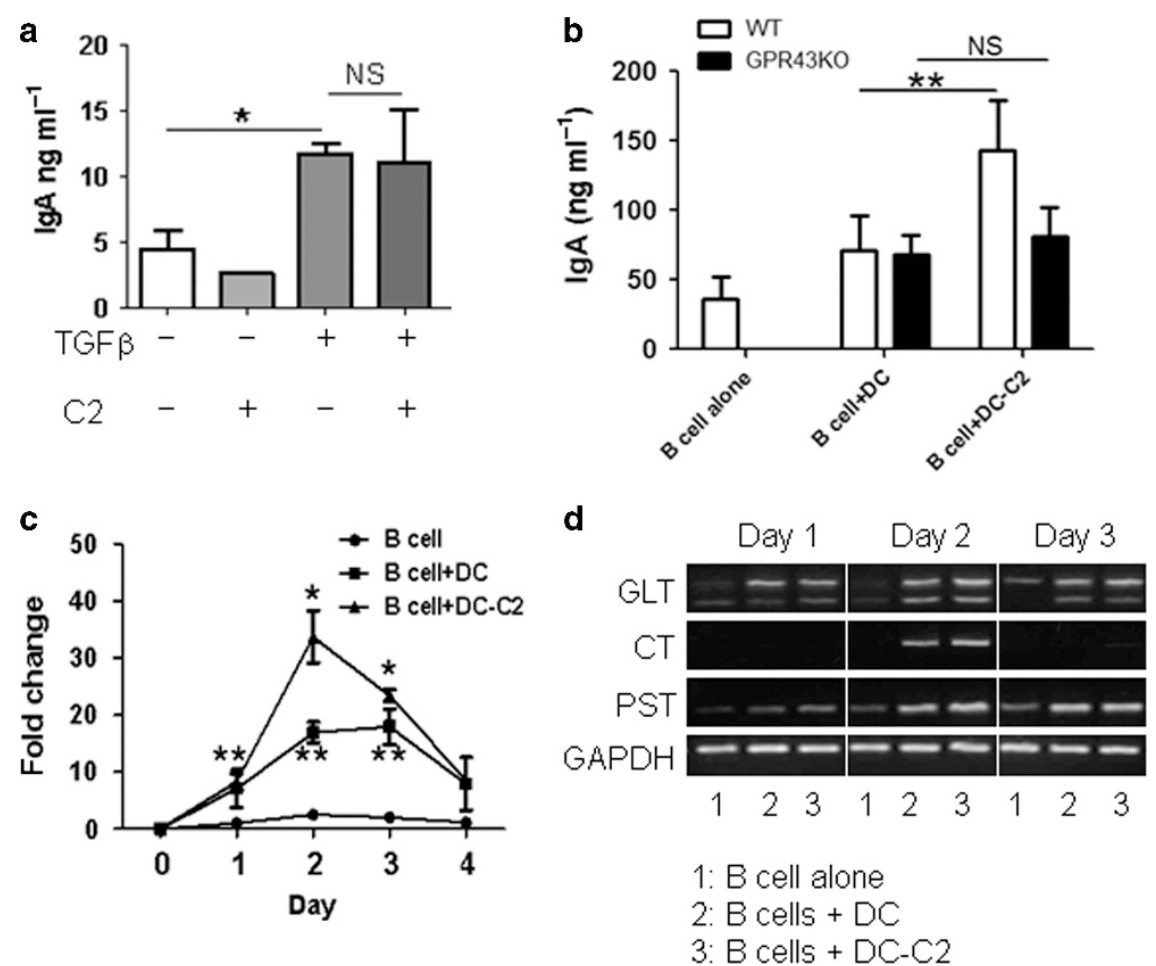

Figure 6 Acetate-treated DCs promote B-cell IgA CSR and IgA production. (a) Splenic naive B cells were cultured with $5 \mu \mathrm{g} \mathrm{ml} \mathrm{I}^{-1}$ anti- $\mu$ and $5 \mu \mathrm{g} \mathrm{ml} \mathrm{I}^{-1}$ CD 40L with or without $1 \mathrm{~mm}$ acetate (C2), $5 \mathrm{ng} \mathrm{ml}^{-1} \mathrm{TGF} \beta, \mathrm{C} 2$ and TGF $\beta$. IgA in supernatants was determined 7 days later by using an ELISA. ${ }^{*} P<0.05$. (b) Splenic DCs from wild type (WT) and GPR43 ${ }^{-1-}$ mice were treated with or without $1 \mathrm{~mm}$ acetate (C2) for $6 \mathrm{~h}$, and then cultured with splenic naive $\mathrm{B}$ cells. IgA in supernatants was determined 7 days later by using ELISA. ${ }^{\star \star} P<0.01$. (c,d) Splenic DCs were treated with or without $1 \mathrm{~mm}$ acetate $(\mathrm{C} 2)$ for $6 \mathrm{~h}$ and then cultured with splenic naive B cells. RNA was collected from cultured B cells on various days during culture and used to analyze molecular markers for IgA class switch recombination. (c) The expression of Aicda was determined by using qRT-PCR and normalized to GAPDH expression. Fold changes were calculated relative to expression of day 0 . ${ }^{\star} P<0.05$ vs B cells with DC. ${ }^{\star \star} P<0.05$ vs B cells alone. (d) $C T$, circular transcripts; GLT, a germline transcripts, and PST, post-switch transcripts, were determined by reverse transcription-PCR (RT-PCR). The data are representative of three to four experiments. 
expression of Aicda (Figure 6c), promoted germ-line production of IgA (GLT) on day 2, and continued to day 3, with CSR to IgA commencing on day 2 (CT). The enhanced transcription of IgA after CSR (PST) was also detected on day 2, in accordance with CSR induction (Figure 6d).

\section{Acetate induces DC production of RA to promote B-cell IgA production}

Several pathways have been identified to mediate DC induction of B-cell IgA production. Intestinal DCs initiate IgA responses by activating $\mathrm{B}$ cells after loading luminal bacteria and producing BAFF/APRIL. ${ }^{5,31}$ TGF $\beta$ is known to induce IgA CSR and maturation. RA, which is enriched in the intestine, has been shown to enhance B-cell IgA production through a mechanism that may affect IgA CSR and the differentiation of
IgA class-switched B cells into IgA-secreting plasma cells. ${ }^{32,33}$ We first investigated whether acetate induces DC production of TGF $\beta$ or expression of BAFF, thus could potentially promote B-cell IgA production. We treated DCs with acetate and assessed TGF $\beta$ production by ELISA and BAFF expression by qRT-PCR. Acetate did not induce DC production of TGF $\beta$ (Figure 7a) or promote DC expression of BAFF (Figure 7b). Addition of TACI-Ig, an inhibitor blocking BAFF/APRIL signaling, to B-cell cultures with acetate-treated DC did not inhibit B-cell IgA production (Figure 7c). Together, these data indicated that DC production of TGF $\beta$ or expression of BAFF is not involved in the acetate induction of $\mathrm{B}$-cell IgA production.

We then investigated whether acetate induced RA production in DC. RA, a metabolite of vitamin A, is produced by retinal dehydrogenases, mainly the aldehyde dehydrogenase family 1 ,
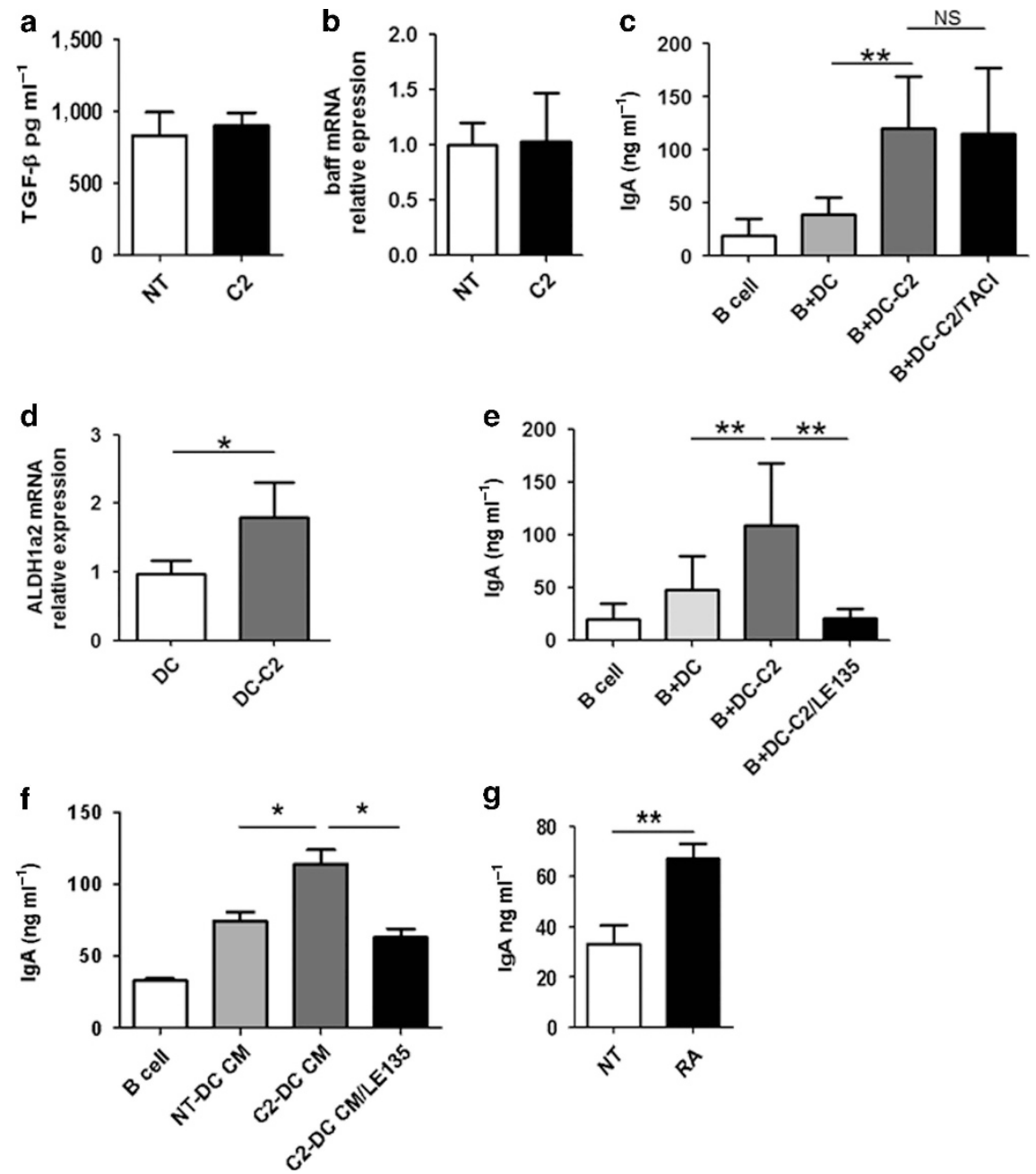

Figure 7 Acetate induces DC expression of Aldh1a2 but not Baff for promotion of B-cell IgA production. Splenic DCs were treated with acetate (C2) for $24 \mathrm{~h}$. (a) The expression of TGF $\beta$ in supernatant was determined by ELISA. (b) The expression of Baff was determined by qRT-PCR. (c) Splenic DCs were pretreated with or without acetate (C2) for $6 \mathrm{~h}$ and then cultured with naive B cells in the presence or absence of $5 \mu \mathrm{g} \mathrm{ml}{ }^{-1} \mathrm{TACl}$. IgA in supernatants was determined 7 days later by using an ELISA. (d) Splenic DCs were treated with acetate (C2) for $24 \mathrm{~h}$, and the expression of Aldh $1 \mathrm{a} 2 \mathrm{was}$ determined by qRT-PCR. (e) Splenic DCs were pretreated with or without acetate (C2) in the presence or absence of $10 \mu \mathrm{m}$ RAR inhibitor LE135 for $6 \mathrm{~h}$ and then cultured with naive B cells. IgA in supernatants was determined 7 days later by using an ELISA. (f) Splenic naive B cells were cultured with $5 \mu \mathrm{g} \mathrm{ml}{ }^{-1}$ anti- $\mu$, $5 \mu \mathrm{g} \mathrm{ml}^{-1} \mathrm{CD} 40 \mathrm{~L}$, and conditioned medium (CM) from splenic DC treated with or without C2, in the presence and absence of LE135. IgA in supernatants was determined 7 days later by using an ELISA. (g) Splenic naive B cells were cultured with $5 \mu \mathrm{g} \mathrm{ml}^{-1}$ anti- $\mu$ and $5 \mu \mathrm{g} \mathrm{ml}{ }^{-1} \mathrm{CD} 40 \mathrm{~L}$ with or without $1 \mu \mathrm{m} \mathrm{RA}$. $\lg A$ in supernatants was determined 7 days later by using an ELISA. The data are representative of $3-4$ experiments. ${ }^{\star} P<0.05 ;{ }^{\star \star} P<0.01$. 
subfamily A1 (ALDH1a1) and A2 (ALDH1a2). ${ }^{34}$ We treated DCs with acetate and determined the expression of ALDH1a1 and ALDH1a2 24h later by qRT-PCR. ALDH1a1 expression was not detected in both acetate-treated DC and control DC (data not shown), whereas expression of Aldh1a2 was significantly increased in acetate-treated DC. (Figure 7d). However, butyrate treatment did not increase the expression of Aldh1a2 of DC (Supplementary Figure S5). To determine whether acetate induction of RA expression enhances B-cell IgA production, we added an antagonist of RAR, receptor for RA, to block RA signaling ${ }^{34}$ in B-cell cultures with acetatetreated DC. Blockade of RAR signaling significantly inhibited B-cell IgA production (Figure 7e). Furthermore, addition of conditioned medium from acetate-treated DC to B-cell cultures promoted IgA production, which was inhibited by blockade of RAR signaling (Figure 7f). To further confirm the role of acetate-induced RA expression in B-cell IgA production, we directly added RA to B-cell cultures with anti- $\mu$ and CD40L. As previously reported, ${ }^{32} \mathrm{RA}$ treatment promoted B-cell IgA production (Figure $7 \mathrm{~g}$ ). Altogether, these data indicated that RA induction in DC by acetate mediates B-cell IgA production.

\section{DISCUSSION}

In this report, we demonstrated that microbiota metabolite acetate promoted intestinal IgA responses to microbiota, which was mediated in part by GPR43. This response reveals a new pathway for microbiota regulation of the host immune response and may be indicative of a novel mechanism for maintenance of intestinal homeostasis. Different from microbiota component TLR ligands, which induce DC expression of BAFF/APRIL through binding of TLRs, which promotes intestinal IgA responses, acetate promotes intestinal IgA responses in part through DC production of RA. Thus, both arms of the microbiota components could cooperatively promote intestinal IgA responses.

The gut microbiota has long been appreciated in the promotion of intestinal IgA responses to microbiota. ${ }^{11,12,35}$ However, the mechanisms by which gut bacteria promote intestinal IgA production and the gut bacterial components mediating microbiota function are still largely unclear. TLR ligands, the gut bacterial components that are sensed through the host cell TLRs, have been shown to trigger several immune responses that are critical for maintaining host microbial homeostasis in the intestines. ${ }^{36}$ TLR-TLR ligand signaling through MyD88 in various cells has been shown to regulate the intestinal IgA response. ${ }^{14,16,17}$ Commensal bacteria-specific IgA was markedly reduced in MyD88 ${ }^{-1-}$ mice. ${ }^{17}$ However, under steady-state conditions, lack of TLR signaling in

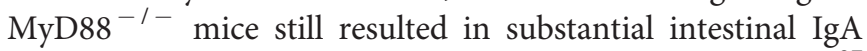
production after colonization with commensal bacteria, ${ }^{37}$ possibly meaning that other components of gut microbiota, in addition to TLR ligands, are able to promote intestinal IgA responses. We now demonstrated that acetate, the metabolite of gut microbiota, can promote the intestinal IgA response to microbiota, which is consistent with a recent report. ${ }^{24}$ Feeding mice with acetate increased intestinal $\operatorname{IgA}^{+}$B cells and $\operatorname{IgA}$ production. Interestingly, acetate also promoted intestinal $\operatorname{Ig} \mathrm{A}$ production in TCR $\beta \times \delta^{-1-}$ mice, suggesting that acetate can induce intestinal IgA in the absence of $\mathrm{T}$ cells. However, as recent studies demonstrated that SCFA promoted the development and function of intestinal Treg cells, ${ }^{20,38}$ which have been shown to regulate intestinal IgA responses, ${ }^{39}$ it is warranted to investigate the role of Treg cells in acetate induction of intestinal IgA responses. Adding acetate directly to B-cell cultures did not induce IgA production. However, acetate induced IgA production when B cells were cultured with DCs, and pretreating DCs with acetate promoted B-cell IgA class switching recombination and IgA production. Our data thereby indicated that acetate induced B-cell IgA production at least in part through DC, but not by directly acting on B cells.

Underneath the epithelium, DCs are at the center of virtually all signaling networks underlying immune protection and homeostasis in the intestinal mucosa. DCs acquire B-celllicensing functions, including IgA-inducing activity, following stimulation by microbiota and various cytokines. ${ }^{5,13,40}$ DCs can sense commensal bacteria by recognizing highly conserved microbial signatures through multiple families of patternrecognition receptors, including TLRs. ${ }^{40}$ In the present study, we demonstrated that DCs can also sense the metabolite SCFA of gut microbiota to regulate the intestinal IgA responses. Intestinal DCs initiate IgA responses by activating B cells after loading luminal bacteria and production of BAFF/APRIL and other factors. ${ }^{5,13,31}$ In addition, gut DCs produce RA in response to microbial stimulation, ${ }^{32}$ which enhances IgA production by increasing IgA CSR and possibly plasma cell differentiation and IgA secretion, as well as by facilitating the migration of IgA class-switched B cells to the gut lamina propria. ${ }^{32,33}$ Our data indicated that acetate did not stimulate DC expression of BAFF and blockade of BAFF/APRIL signaling by TACI, an inhibitor of BAFF/APRIL signaling, did not affect the acetate induction of B-cell IgA production. In contrast, acetate induced RA production in DC, and the blockade of RA signaling inhibited acetate induction of B-cell IgA production. It is currently unknown how acetate induces DC production of RA. Our preliminary data indicated that acetate also activated DC mama mechanistic target of rapamycin (mTOR) pathway and blockade of mTOR inhibited acetate induction of B-cell IgA production (data not shown), indicating that acetate signals through the mTOR pathway in DC to promote B-cell IgA production. We are currently investigating whether acetateactivation of DC mTOR pathway mediates RA production.

These data thereby suggested to us that acetate induction of RA in DC, but not BAFF/APRIL, at least in part, mediates promotion of IgA production. As gut microbiota can stimulate DC expression of BAFF/APRIL through TLR-TLR ligand interaction, it is very likely that both components of microbiota, namely TLR ligands and SCFA, promote intestinal IgA production in a cooperative manner.

SCFA have been shown to regulate host immune response through intracellular targets such as histone deacetylases (HDAC) $)^{41-43}$ or by binding cell-surface receptors such as GPR41, GPR43 and GPR109 $\alpha^{43,44}$ Although propionate and 
butyrate are the most potent agonists for GPR41, but weak agonists for GPR43, acetate is the potent agonist for GPR43, but a weak agonist for GPR $41 .{ }^{25}$ SCFA-GPR43 interaction has been implicated in regulating intestinal inflammatory responses and other inflammatory diseases. ${ }^{45} \mathrm{GPR} 43^{-1-}$ mice develop more severe inflammation in animal models of colitis upon inflammatory insults, ${ }^{26}$ an indicator of the importance of SCFA-GPR43 interaction for maintenance of intestinal homeostasis. Deficiency in SCFA-GPR43 interaction also leads to exacerbated or unresolved inflammation in animal models of arthritis and asthma, ${ }^{26}$ which may be the consequence of broken intestinal homeostasis as well. A recent study further demonstrated that SCFA activated the NLRP3 inflammasome in IEC by the binding of GPR43 and GPR109a, which contributed to its protection against intestinal inflammation. ${ }^{44}$ More recently, dietary fiber has been shown to enhance oral tolerance, probably through induction of intestinal IgA, which was dependent on GPR43 and GPR $41,{ }^{23}$ suggesting that GPR43 and GPR41 could mediate high dietary fiber-induction of intestinal IgA. Our current study demonstrated that GPR43 mediated the acetate induction of intestinal IgA response to microbiota, in that GPR43 ${ }^{-1-}$ mice demonstrated lower IgA production and decreased numbers of $\operatorname{IgA}^{+} \mathrm{B}$ cells in the intestines. Although both acetate and butyrate binds GPR43, the affinity of binding by acetate is much higher than that of butyrate. Feeding with acetate promoted intestinal IgA in WT but not GPR $43^{-1-}$ mice, however, feeding with butyrate did not promote intestinal IgA. While acetate-treated WT DCs promoted B-cell IgA production, acetate-treated GPR43 ${ }^{-1-}$ DCs failed to do so. As SCFA propionate also binds GPR43, even at a low affinity, we are actively investigating whether it also promotes intestinal IgA responses. Although it has been shown that SCFA inhibit histone deacetylases in a GPR41- and GPR43-independent manner, a recent report also suggested that HDAC activity could be regulated in a GPR43-dependent manner. ${ }^{20,46}$ We are still unclear at this point as to whether HDAC inhibitory activity of acetate is involved in the regulation of the intestinal $\operatorname{IgA}$ responses.

\section{METHODS}

Mouse models and housing conditions. C57BL/6 (WT) mice and $\mathrm{TCR} \beta \mathrm{x} \delta^{-1-}$ mice were purchased from Jackson Laboratory. $\mathrm{GPR}^{-1-}$ (Ffar2 $\left.^{\text {tm1Lex }}\right)$ mice were obtained from Bristol-Myers Squibb. All of the mice strains were housed and maintained in the animal resource center of the University of Texas Medical Branch. The littermates of WT and GPR43 ${ }^{-1-}$ mice from breeding pairs of GPR $43^{+/}$mice were used as indicated in text. Female mice between 6 and 12 weeks were used for all experiments. All experiments were reviewed and approved by the Institutional Animal Care and Use Committees of the University of Texas Medical Branch. All the methods were carried out in accordance with the approved guidelines.

Reagents and antibodies. The following antibodies were used for flow cytometry: Anti-CD19 and anti-IgA antibodies were purchased from Biolegends (San Diego, CA, USA). The Live/Dead Fixable Dead Cell Stain Kit was purchased from Life Technologies (Carlsbad, CA, USA). RAR inhibitor LE135 from Tocris Bioscience (Ellisville, MO, USA), and acetate and butyrate from Sigma. All-trans-RA was purchased from Sigma-Aldrich (St. Louis, MO, USA), TGF $\beta$ from
Biolegends (San Diego, CA, USA), anti- $\mu$ from Jackson ImmunoResearch Laboratories (West Grove, PA, USA), and CD40L from BioXCell (West Lebanon, NH, USA).

SCFA treatment. Firstly, the mice were given $1 \mathrm{gl}^{-1}$ metronidazole (Sigma-Aldrich), $0.5 \mathrm{gl}^{-1}$ vancomycin (Hospira), $1 \mathrm{gl}^{-1}$ ampicillin (Sigma-Aldrich) and $1 \mathrm{gl}^{-1}$ kanamycin (Fisher Scientific) in drinking water for 10 days. Acetate and butyrate were added separately to drinking water containing antibiotics at $300 \mathrm{~mm}$ and $\mathrm{pH}$-adjusted as needed.

Fecal pellet collection. Fecal pellets were collected, weighed, and placed in PBS with $20 \mathrm{~mm}$ EDTA, $2 \mathrm{~mm}$ PMSF and $0.04 \mathrm{mg} \mathrm{ml}^{-1}$ soybean trypsin inhibitor. As described previously, ${ }^{29}$ the mixtures were centrifuged to remove bacteria and insoluble debris. The supernatants were collected and stored at $-20{ }^{\circ} \mathrm{C}$.

Th17 generation. As described previously, ${ }^{29} \mathrm{CD} 4^{+} \mathrm{T}$ cells were isolated from the spleens of WT and GPR $43^{-1-}$ mice by using antimouse CD4-magnetic beads (BD Biosciences, San Jose, CA). To polarize Th17 cells, CD4 ${ }^{+} \mathrm{T}$ cells were cultured with $10 \mathrm{ng} \mathrm{ml}^{-1}$ TGF- $\beta 1,30 \mathrm{ng} \mathrm{ml}^{-1}$ interleukin (IL) $-6,10 \mu \mathrm{g} \mathrm{ml}^{-1}$ anti-IFN- $\gamma$, and $10 \mu \mathrm{g} \mathrm{ml}^{-1}$ anti-IL-4 and activated with anti-CD3 and anti-CD28 for $5 \mathrm{~d}$.

LPMC isolation. As described previously, ${ }^{29}$ intestines were removed, sliced, and digested with Collagense IV and DNase. The cells were resuspended in 40\% Percoll and carefully overlaid onto 75\% Percoll. The interface containing the lamina propria lymphocytes was collected.

Splenic DC isolation. Splenic DCs were isolated by using MACS DC isolation kits (Miltenyi Biotec, San Diego, CA).

Generation of DC conditioned medium. Splenic DCs were isolated and treated with or without $1 \mathrm{~mm}$ acetate (C2) for 2 days. The supernatant was collected at day 2 to serve as conditioned medium (CM). CM was added to B-cell cultures at 1:1 ratio with fresh culture media.

B-cell culture. Naive $\operatorname{IgD}^{+}$B cells were isolated from spleens by using MACS isolation kits (Miltenyi Biotec San Diego, CA, USA) and cultured with anti- $\mu, \mathrm{CD} 40 \mathrm{~L}$, or DCs as indicated in text. The cells were harvested for isolation of RNA, as indicated in the text, or culture supernatants collected at days 5-7 to measure IgA.

Isolation of IEC. The intestines were cut into small pieces $(<1 \mathrm{~cm})$ and incubated in HBSS buffer containing $5 \% \mathrm{FBS}$ at $37^{\circ} \mathrm{C}$ for 40 min with $5 \mathrm{~mm}$ EDTA. After passaging through a $100-\mu \mathrm{M}$ cell strainer (BD Falcon), the cells were separated from a $20 \% / 40 \%$ Percoll interface (Amersham Pharmacia Biotech). The cell viability was normally $>90 \%$.

Flow cytometric analysis. Fluorescence-activated cell sorting (FACS) was performed as previously described. ${ }^{29}$ Briefly, after washing with $\mathrm{PBS}$, the cells were incubated with various conjugated mAbs, washed, and fixed in $1 \%$ buffered paraformaldehyde, and quantitated by using an LSRII/Fortessa and FACSDiva software (Becton Dickinson, Mountain View, CA, USA). Data analysis was carried out by using FlowJo. A mAb of the same isotype but irrelevant specificity was used as a negative control.

Measurement of IgA-coating gut bacteria. As described previously, ${ }^{9}$ fecal pellets were collected from the co-housed mice and homogenized by bead beating. The supernatants containing fecal bacteria were collected and washed with $1 \mathrm{ml}$ PBS containing $1 \%$ bovine serum albumin. After incubation with blocking buffer, the fecal homogenates were stained with PE-conjugated Anti-Mouse IgA and Live/dead dye for $30 \mathrm{~min}$ on ice.

ELISAs. Ninety-six-well plates were coated with $1 \mathrm{mg} \mathrm{ml}^{-1}$ anti-IgA at $4{ }^{\circ} \mathrm{C}$ overnight. Fecal samples were diluted $1 / 200$. Usually a twofold 
serial dilution was made. Samples were incubated at room temperature for $2 \mathrm{~h}$, and biotinylated anti-IgA was added. One hour later, HRPconjugated streptavidin was added. The plates were developed by using TMB substrate and analyzed at $450 \mathrm{~nm}$ according to the manufacturer's instructions.

Quantitative real-time PCR (qRT-PCR). Total RNA was extracted with TRIzol (Invitrogen, Carlsbad, CA) and followed by cDNA synthesis with reverse transcriptase. qPCR was performed by using TaqMan Gene Expression Assays. Predesigned primers and probes for Pigr, Baff, Aldh1a1, Aldh1a2, Aicda, and Gapdh were ordered from Applied Biosystems, and the data were normalized to Gapdh mRNA expression. The primers I $\alpha$ F (5'-CCAGGCATGGTTGAGATAGAG ATAG- $\left.3^{\prime}\right)$ and $C \alpha R\left(5^{\prime}\right.$-GAGCTGGTGGGAGTGTCAGTG-3') were used to detect germ-line $\alpha$ transcripts. ${ }^{47}$ Circular transcripts were detected by a nested PCR by using the outer primers $\mathrm{I} \mu 4$ $\left(5^{\prime}\right.$-ACCCTGGATGACTTCAGTGT-3') and I $\alpha$ up4 (5'-CATCTGG ACTCCTCTGCTCA $-3^{\prime}$ ) and followed by the inner primers $\mathrm{I} \alpha \mathrm{F}$ (5'-CCAGGCATGGTTGAGATAGAGATAG- $3^{\prime}$ ) and C $\mu \mathrm{R}\left(5^{\prime}\right.$-AAT GGTGCTGGGCAGGAAGT- $\left.3^{\prime}\right) .{ }^{47}$ Post-switch $\alpha$ transcripts were detected with the primers $\mathrm{I} \mu \mathrm{F}$ ( $5^{\prime}$-GAGCTGGTGGGAGTGTCA GTG $\left.-3^{\prime}\right)^{47}$ and $\mathrm{C} \alpha \mathrm{R}$. PCR products were visualized by electrophoresis on $1.5 \%$ agarose gels

Statistical analysis. Student's $t$-test in Prism 5.0 (Graphpad, San Diego, CA) was used to determine levels of significance for comparisons between samples. Mean \pm s.d. is represented on graphs. $P$-values of $<0.05$ were considered to be statistically significant. ${ }^{\star} P<0.05 ;{ }^{*} P<0.01$.

SUPPLEMENTARY MATERIAL is linked to the online version of the paper at http://www.nature.com/mi

\section{ACKNOWLEDGMENTS}

This work was supported by NIH grants DK098370 and DK105585, and John Sealy Memorial Endowment Fund. We appreciate Ms. Mardelle Susman of The University of Texas Medical Branch for proofreading the manuscript.

\section{AUTHOR CONTRIBUTIONS}

W.W. and M.S. performed experiments, analyzed the data and wrote the manuscript. F.C., A.T.C., H.L., Y.Z., X.H., Y.X., and S.Y. performed part of experiments, analyzed the data and reviewed the manuscript. Q.Z. provided the reagents and revised the manuscript. Z.L. designed the project and revised the manuscript. Y.C. conceived the project, designed the experiments, and wrote the manuscript.

\section{DISCLOSURE}

The authors declared no conflict of interest.

co 2017 Society for Mucosal Immunology

\section{REFERENCES}

1. Sansonetti, P.J. War and peace at mucosal surfaces. Nat. Rev. Immunol. 4, 953-964 (2004).

2. Mazmanian, S.K., Round, J.L. \& Kasper, D.L. A microbial symbiosis factor prevents intestinal inflammatory disease. Nature 453, 620-625 (2008).

3. Fagarasan, S., Kawamoto, S., Kanagawa, O. \& Suzuki, K. Adaptive immune regulation in the gut: $T$ cell-dependent and T cell-independent IgA synthesis. Annu. Rev. Immuno./ 28, 243-273 (2010).

4. Macpherson, A.J., McCoy, K.D., Johansen, F.E. \& Brandtzaeg, P. The immune geography of IgA induction and function. Mucosal Immunol 1, 11-22 (2008).

5. Cerutti, A. \& Rescigno, M. The biology of intestinal immunoglobulin A responses. Immunity 28, 740-750 (2008).

6. Peterson, D.A., McNulty, N.P., Guruge, J.L. \& Gordon, J.I. IgA response to symbiotic bacteria as a mediator of gut homeostasis. Cell Host Microbe 2, 328-339 (2007).
7. Suzuki, K. et al. Aberrant expansion of segmented filamentous bacteria in IgA-deficient gut. Proc. Natl Acad. Sci. USA 101, 1981-1986 (2004).

8. Bunker, J.J. et al. Innate and adaptive humoral responses coat distinct commensal bacteria with immunoglobulin A. Immunity 43, 541-553 (2015).

9. Palm, N.W. et al. Immunoglobulin A coating identifies colitogenic bacteria in inflammatory bowel disease. Cell 158, 1000-1010 (2014).

10. Murthy, A.K., Dubose, C.N., Banas, J.A., Coalson, J.J. \& Arulanandam, B.P. Contribution of polymeric immunoglobulin receptor to regulation of intestinal inflammation in dextran sulfate sodium-induced colitis. J. Gastroenterol. Hepatol. 21, 1372-1380 (2006).

11. Crabbe, P.A., Bazin, H., Eyssen, H. \& Heremans, J.F. The normal microbial flora as a major stimulus for proliferation of plasma cells synthesizing IgA in the gut. The germ-free intestinal tract. Int. Arch. Allergy Appl. Immunol. 34, 362-375 (1968).

12. Benveniste, J., Lespinats, G. \& Salomon, J. Serum and secretory IgA in axenic and holoxenic mice. J. Immunol. 107, 1656-1662 (1971).

13. Cerutti, A. The regulation of IgA class switching. Nat. Rev. Immunol. 8, 421-434 (2008).

14. He, B. et al. Intestinal bacteria trigger T cell-independent immunoglobulin A(2) class switching by inducing epithelial-cell secretion of the cytokine APRIL. Immunity 26, 812-826 (2007).

15. Tezuka, H. et al. Prominent role for plasmacytoid dendritic cells in mucosal T cell-independent IgA induction. Immunity 34, 247-257 (2011).

16. Kubinak, J.L. et al. MyD88 signaling in T cells directs IgA-mediated control of the microbiota to promote health. Cell Host Microbe 17, 153-163 (2015).

17. Kirkland, D. et al. B cell-intrinsic MyD88 signaling prevents the lethal dissemination of commensal bacteria during colonic damage. Immunity 36, 228-238 (2012).

18. Haghikia, A. et al. Dietary fatty acids directly impact central nervous system autoimmunity via the small intestine. Immunity 43, 817-829 (2015).

19. Macia, L. et al. Microbial influences on epithelial integrity and immune function as a basis for inflammatory diseases. Immunol. Rev. 245, 164-176 (2012).

20. Smith, P.M. et al. The microbial metabolites, short-chain fatty acids, regulate colonic Treg cell homeostasis. Science 341, 569-573 (2013).

21. Cummings, J.H., Pomare, E.W., Branch, W.J., Naylor, C.P. \& Macfarlane, G.T. Short chain fatty acids in human large intestine, portal, hepatic and venous blood. Gut 28, 1221-1227 (1987).

22. Trompette, A. et al. Gut microbiota metabolism of dietary fiber influences allergic airway disease and hematopoiesis. Nat. Med. 20, 159-166 (2014)

23. Tan, J. et al. Dietary fiber and bacterial SCFA enhance oral tolerance and protect against food allergy through diverse cellular pathways. Cell Rep. 15, 2809-2824 (2016).

24. Kim, M., Qie, Y., Park, J. \& Kim, C.H. Gut Microbial Metabolites Fuel Host Antibody Responses. Cell Host Microbe 20, 202-214 (2016).

25. Brown, A.J. et al. The Orphan G protein-coupled receptors GPR41 and GPR43 are activated by propionate and other short chain carboxylic acids. J. Biol. Chem. 278, 11312-11319 (2003).

26. Maslowski, K.M. et al. Regulation of inflammatory responses by gut microbiota and chemoattractant receptor GPR43. Nature 461, 1282-1286 (2009).

27. Phalipon, A. \& Corthesy, B. Novel functions of the polymeric lg receptor: well beyond transport of immunoglobulins. Trends Immunol. 24, 55-58 (2003).

28. Milligan, G., Stoddart, L.A. \& Smith, N.J. Agonism and allosterism: the pharmacology of the free fatty acid receptors FFA2 and FFA3. Br. J. Pharmacol. 158, 146-153 (2009).

29. Cao, A.T., Yao, S., Gong, B., Elson, C.O. \& Cong, Y. Th17 cells upregulate polymeric Ig receptor and intestinal IgA and contribute to intestinal homeostasis. J. Immunol. 189, 4666-4673 (2012).

30. Kinoshita, K., Harigai, M., Fagarasan, S., Muramatsu, M. \& Honjo, T. A hallmark of active class switch recombination: transcripts directed by I promoters on looped-out circular DNAs. Proc. Natl Acad. Sci. USA 98, 12620-12623 (2001).

31. Litinskiy, M.B. et al. DCs induce CD40-independent immunoglobulin class switching through BLyS and APRIL. Nat. Immunol. 3, 822-829 (2002). 


\section{ARTICLES}

32. Mora, J.R. et al. Generation of gut-homing IgA-secreting B cells by intestinal dendritic cells. Science 314, 1157-1160 (2006).

33. Watanabe, K. et al. Requirement for Runx proteins in IgA class switching acting downstream of TGF-beta 1 and retinoic acid signaling. J. Immunol. 184, 2785-2792 (2010).

34. Feng, T., Cong, Y., Qin, H., Benveniste, E.N. \& Elson, C.O. Generation of mucosal dendritic cells from bone marrow reveals a critical role of retinoic acid. J. Immunol. 185, 5915-5925 (2010).

35. Macpherson, A.J., Gatto, D., Sainsbury, E., Harriman, G.R., Hengartner, H. \& Zinkernagel, R.M. A primitive Tcell-independent mechanism of intestinal mucosal IgA responses to commensal bacteria. Science $\mathbf{2 8 8 ,} 2222-2226$ (2000).

36. Blander, J.M. \& Medzhitov, R. Regulation of phagosome maturation by signals from toll-like receptors. Science 304, 1014-1018 (2004).

37. Slack, E. et al. Innate and adaptive immunity cooperate flexibly to maintain host-microbiota mutualism. Science 325, 617-620 (2009).

38. Arpaia, N. et al. Metabolites produced by commensal bacteria promote peripheral regulatory T-cell generation. Nature 504, 451-455 (2013).

39. Cong, Y., Feng, T., Fujihashi, K., Schoeb, T.R. \& Elson, C.O. A dominant, coordinated $T$ regulatory cell-IgA response to the intestinal microbiota. Proc. Natl Acad. Sci. USA 106, 19256-19261 (2009).

40. Kelsall, B.L. \& Rescigno, M. Mucosal dendritic cells in immunity and inflammation. Nat. Immunol. 5, 1091-1095 (2004).
41. Chang, P.V., Hao, L., Offermanns, S. \& Medzhitov, R. The microbial metabolite butyrate regulates intestinal macrophage function via histone deacetylase inhibition. Proc. Natl Acad. Sci. USA 111, 2247-2252 (2014).

42. Ganapathy, V., Thangaraju, M., Prasad, P.D., Martin, P.M. \& Singh, N. Transporters and receptors for short-chain fatty acids as the molecular link between colonic bacteria and the host. Curr. Opin. Pharmacol. 13, 869-874 (2013).

43. Kim, C.H., Park, J. \& Kim, M. Gut microbiota-derived short-chain Fatty acids, T cells, and inflammation. Immune Netw. 14, 277-288 (2014).

44. Macia, L. et al. Metabolite-sensing receptors GPR43 and GPR109A facilitate dietary fibre-induced gut homeostasis through regulation of the inflammasome. Nat. Commun. 6, 6734 (2015).

45. McKenzie, C.I., Mackay, C.R. \& Macia, L. GPR43 - A prototypic metabolite sensor linking metabolic and inflammatory diseases. Trends Endocrinol. Metab. 26, 511-512 (2015).

46. Park, J. et al. Short-chain fatty acids induce both effector and regulatory $T$ cells by suppression of histone deacetylases and regulation of the mTORS6K pathway. Mucosal Immunol. 8, 80-93 (2015).

47. Bergqvist, P., Gardby, E., Stensson, A., Bemark, M. \& Lycke, N.Y. Gut IgA class switch recombination in the absence of CD40 does not occur in the lamina propria and is independent of germinal centers. J. Immunol. 177, 7772-7783 (2006). 